\title{
Negative myoclonus associated with tramadol use
}

\author{
Seong Yoon $\mathrm{Bae}^{1}$, Se-Jin $\mathrm{Lee}^{2}$ \\ ${ }^{1}$ Department of Neurology, Daegu Fatima Hospital, Daegu, Korea \\ ${ }^{2}$ Department of Neurology, Yeungnam University College of Medicine, Daegu, Korea
}

Received: March 2, 2020

Revised: March 29, 2020

Accepted: April 1, 2020

Corresponding author:

Se-Jin Lee

Department of Neurology, Yeungnam University College of Medicine, 170 Hyeonchung-ro Nam-gu, Daegu 42415, Korea

Tel: +82-53-620-3683

Fax: +82-53-627-1688

E-mail: sejinmayo@ynu.ac.kr
Negative myoclonus (NM) is a shock-like jerky involuntary movement caused by a sudden, brief interruption of tonic muscle contraction. NM is observed in patients diagnosed with epilepsy, metabolic encephalopathy, and drug toxicity and in patients with brain lesions. A 55-year-old man presented with NM in both his arms and neck. He has taken medications containing tramadol at a dose of 80-140 mg/day for 5 days due to common cold. He had no history of seizures. Acute lesions were not observed during magnetic resonance imaging, and abnormal findings in his laboratory tests were not noted. His NM resolved completely after the discontinuation of tramadol and the oral administration of clonazepam. Our case report suggests that tramadol can cause NM in patients without seizure history or metabolic disorders, even within its therapeutic dose.

Keywords: Negative myoclonus; Myoclonus; Seizure; Tramadol

\section{Introduction}

Negative myoclonus (NM) is a brief involuntary jerky movement caused by a sudden lapse of tonic muscle contraction in the affected body segments. NM may appear not only in normal individuals physiologically during fear or sleep transition but also in patients with metabolic encephalopathy as flapping tremor (asterixis) [1-3]. Anticonvulsants, such as gabapentin and pregabalin, may also induce NM [4]. Tramadol, a synthetic codeine, is an analgesic used to treat various types of pain and is known to provoke generalized tonic-clonic seizure or myoclonus [5-8]. However, based on the Korean literature, studies assessing the presence of $\mathrm{NM}$ due to tramadol use have not been conducted yet. We hereby report a case of NM caused by the therapeutic dose of tramadol.

\section{Case}

This study was approved by the Institutional Review Board (IRB) of the Yeungnam University Hospital (IRB No: 2020-04-010).

A 55-year-old man visited the emergency room following the complaint of sudden involuntary movements of both his arms and neck at night. When he was raising his arms to wash his face or to hold a light object, his arms abruptly lapsed and jerked downward. His symptoms progressed overnight. When he tried to take a posture of holding a brush to practice calligraphy, his right arm twitched and jerked downward; thus, he pointed the paper repetitively with brush. When he tried to raise his head upright, his head jerked backward.

He has taken medications containing tramadol, acetaminophen, acetylcysteine, and prednisolone for 5 days as a remedy to common cold. The daily dose of tramadol administered was approximately $80-140 \mathrm{mg}$. He has been taking aspirin and atorvastatin for 3 years due to asymptomatic cerebral infarction and dyslipidemia. He had no history of seizures, and his chronic hepatitis $B$ was treated 4 years ago. He was a light drinker and had not consumed alcohol for 2 weeks prior to taking the medications due to

Copyright (C) 2020 Yeungnam University College of Medicine

This is an Open Access article distributed under the terms of the Creative Commons Attribution Non-Commercial License (http://creativecommons.org/licenses/by-nc/4.0/) which permits unrestricted non-commercial use, distribution, and reproduction in any medium, provided the original work is properly cited. 
common cold.

When he arrived at the hospital, his vital signs were unremarkable. The patient was alert and appeared normal during his cranial nerve examination and motor and sensory function tests without pathologic reflexes. When he stretched both arms forward with dorsiflexion of the wrists in the sitting position, NM was provoked irregularly every 3 seconds, and it was not observed at rest. While he was extending his head upright, NM was also provoked on the head at a lower frequency compared with that of his arms.

There were no abnormal findings in complete blood cell count, urinalysis, and liver function, electrolyte, kidney function, blood ammonia concentration, or thyroid function test. Acute lesion was not observed on brain magnetic resonance imaging (MRI).

We presumed this case as NM associated with tramadol based on patient's medical history, laboratory tests, and brain MRI. We discontinued the patient's medications including tramadol and prescribed oral clonazepam $0.5 \mathrm{mg}$ once. The following morning, his symptoms completely improved, and electroencephalography (EEG) revealed no abnormal findings. He was discharged on the third day of admission and had no recurrence of NM for 3 years.

\section{Discussion}

Tramadol is a widely used analgesic for acute and chronic pain because it is less likely to be abused and has fewer side effects including respiratory depression than other opioid analgesics [9]. The maximum recommended daily dose of tramadol is $400 \mathrm{mg}$. It is well known that tramadol can provoke seizure and myoclonus within its therapeutic doses $[5,7,8]$.

A French epidemiologic study on the incidence of drug-induced myoclonus reported that $12 \%$ of drug-induced myoclonus was caused by opioid agents, $25 \%$ of them were due to tramadol [5]. The incidence of tramadol-induced NM is possibly underestimated considering the presence of mild and transient symptoms observed in substantial patients after taking tramadol.

Tramadol acts as a weak mu-opioid receptor agonist and inhibits $\gamma$-aminobutyric acid receptor; it can eventually provoke seizure and myoclonus [10]. Furthermore, tramadol inhibits the reuptake of serotonin at the synaptic cleft and increases the concentration of serotonin in the synaptic cleft. It has been reported that the serotonin neurotransmitter system plays a role in the development of gabapentin-induced myoclonus [11-13]. These are the wellknown pathogenic mechanisms of myoclonus.

Patient with NM cannot sustain a posture because the affected limbs or neck are suddenly lapse and jerk downward by brief interruption of tonic muscle contraction. NM does not occur at rest, while positive myoclonus may appear at rest or during volun- tary movement. Our patient developed sudden, brief, involuntary, and shock-like jerky movements while keeping of certain postures, such as elevating his arms forward and extending his neck. However, these symptoms completely disappeared at rest. On neurological examination, these movements were easily reproduced during sustained postures, which were irregular, repetitive, and symmetric. These clinical features were consistent with NM.

In our case, the patient suddenly showed NM after taking tramadol and dramatically recovered by the administration of clonazepam and discontinuation of tramadol. He had no history of seizure or myoclonus. Furthermore, there were no significant abnormal findings in the laboratory tests or brain MRI. Therefore, we concluded this case as NM associated with tramadol use.

There have been several case reports and case series of drug-induced NM, including gabapentin and pregabalin $[4,14]$. Drug-induced NM is usually believed to be of subcortical origin because of the absence of cortical correlates of NM in the electrophysiological tests $[2,15]$. In our case, the characteristics of NM were similar to those of asterixis in that it was multifocal, bilateral, and symmetric and occurred without any external stimuli. Asterixis, the most characteristic subtype of NM, is known to be of subcortical origin and can occur in drug-induced disorders and toxic metabolic encephalopathies [2].

In general, subcortical NM shows good response to clonazepam as in our case. Although we did not perform simultaneous EEG-electromyography or somatosensory evoked potential test because the patient's symptoms improved completely after the administration of clonazepam, we suggest that this case is likely to be $\mathrm{NM}$ of subcortical origin.

Clinicians must be significantly cautious of the possible occurrence of myoclonus or seizure in patients taking tramadol. We suggest that tramadol can potentially induce an $\mathrm{NM}$, even in patients taking tramadol within its therapeutic dose and without history of seizure or myoclonus.

\section{Acknowledgments}

\section{Conflicts of interest}

No potential conflict of interest relevant to this article was reported.

\section{Author contributions}

Conceptualization: SYB, SL; Data curation: SYB; Formal analysis: SL; Investigation: SYB; Supervision, Validation: SL; Writing-original draft: SYB; Writing-review \& editing: SL. 


\section{ORCID}

\section{Seong Yoon Bae, https://orcid.org/0000-0003-1592-3601}

Se-Jin Lee, https://orcid.org/0000-0001-7438-691X

\section{References}

1. Rubboli G, Tassinari CA. Negative myoclonus. An overview of its clinical features, pathophysiological mechanisms, and management. Neurophysiol Clin 2006;36:337-43.

2. Kojovic M, Cordivari C, Bhatia K. Myoclonic disorders: a practical approach for diagnosis and treatment. Ther Adv Neurol Disord 2011;4:47-62.

3. Eberhardt O, Topka H. Myoclonic disorders. Brain Sci 2017; $7: 103$.

4. Kim JB, Jung JM, Park MH, Lee EJ, Kwon DY. Negative myoclonus induced by gabapentin and pregabalin: a case series and systematic literature review. J Neurol Sci 2017;382:36-9.

5. Brefel-Courbon C, Gardette V, Ory F, Montastruc JL. Drug-induced myoclonus: a French pharmacovigilance database study. Neurophysiol Clin 2006;36:333-6.

6. Jovanovic-Cupic V, Martinovic Z, Nesic N. Seizures associated with intoxication and abuse of tramadol. Clin Toxicol (Phila) 2006;44:143-6.

7. Talaie H, Panahandeh R, Fayaznouri M, Asadi Z, Abdollahi M.
Dose-independent occurrence of seizure with tramadol. J Med Toxicol 2009;5:63-7.

8. Boostani R, Derakhshan S. Tramadol induced seizure: a 3-year study. Caspian J Intern Med 2012;3:484-7.

9. Grond S, Sablotzki A. Clinical pharmacology of tramadol. Clin Pharmacokinet 2004;43:879-923.

10. Rehni AK, Singh I, Kumar M. Tramadol-induced seizurogenic effect: a possible role of opioid-dependent gamma-aminobutyric acid inhibitory pathway. Basic Clin Pharmacol Toxicol 2008; 103:262-6.

11. Pappert EJ, Goetz CG, Stebbins GT, Belden M, Carvey PM. 5-Hydroxytryptophan-induced myoclonus in guinea pigs: mediation through 5-HT1/2 receptor subtypes. Eur J Pharmacol 1998;347:51-6.

12. Pranzatelli MR. Serotonin and human myoclonus. Rationale for the use of serotonin receptor agonists and antagonists. Arch Neurol 1994;51:605-17.

13. Asconape J, Diedrich A, DellaBadia J. Myoclonus associated with the use of gabapentin. Epilepsia 2000;41:479-81.

14. Park KD, Kim MK, Lee SJ. Negative myoclonus associated with pregabalin. Yeungnam Univ J Med 2018;35:240-3.

15. Li JY, Cunic DI, Paradiso G, Gunraj C, Pal PK, Lang AE, et al. Electrophysiological features of myoclonus-dystonia. Mov Disord 2008;23:2055-61. 